\title{
Un nouveau départ pour notre revue
}

Avec ce premier numéro, notre revue change. Tout d'abord son utilisation : vous continuerez à la lire comme d'habitude, dans votre bureau, chez vous, dans le train, vous pouvez même en faire un livre de chevet, et maintenant vous pouvez aussi la consulter via Internet. En vous connectant à :

\section{http://www.edpsciences.org/radio}

vous accédez directement à la page d'accueil ${ }^{1}$ de la revue. Vous continuerez à pourvoir consulter les (nouvelles) instructions aux auteurs, la politique éditoriale de la revue, et toute information relative à la vie de la revue.

À partir de ce numéro, vous aurez également accès à l'intégralité des articles, aux rubriques revue des livres, revue des thèses, etc. au format PDF. Un lien, situé en bas de la table des matières, vous permettra d'accéder au RP magazine via le site de la SFRP (www.sfrp.asso.fr). De plus, grâce au système d'indexation «CrossRef », les références de chaque article (à condition de citer des revues internationales) seront « cliquables » : vous pourrez accéder directement à l'article cité. Nous demandons donc à nos auteurs de bien veiller aux références qu'ils utilisent pour que le système fonctionne au mieux.

D'autre part, pour faciliter les recherches sur Internet, nous demandons à chaque auteur d'attribuer des mots clés à son article (ce qui change un peu nos habitudes). Une liste de mots clés est proposée sur le site d'EDP Sciences.

Pour avoir accès à la version «en ligne », les membres de la SFRP recevront un mot de passe qu'ils pourront utiliser. Les abonnés non-membres devront contacter directement l'éditeur pour connaître les modalités d'enregistrement (subscribers@edpsciences.org).

Enfin, n'oubliez pas de vous inscrire à l'e-mail alert pour recevoir gratuitement la table des matières « interactive » dès la mise en ligne du numéro (environ un mois avant la distribution du volume papier).

Néanmoins, tout ceci se fait dans un contexte budgétaire difficile, alors nous avons décidé d'utiliser au maximum l'informatique : vous pourrez soumettre vos articles au format PDF et vous recevrez des tirés-à-part uniquement au format PDF. Le courrier classique demeurera, mais il devrait être considérablement réduit et réservé à quelques cas particuliers.

Ce premier numéro est aussi l'occasion de souligner que nous avons de plus en plus d'auteurs hors de France, cette tendance est à poursuivre, elle montre tout

\footnotetext{
1 Celle-ci est décrite dans la rubrique Internet Écl@ire.

DOI: $10.1051 /$ radiopro200505

RADIOPROTECTION - VOL. 40 - $\mathrm{N}^{\circ} 1$ (2005)
} 
ÉDITORIAL

simplement que notre revue commence une percée internationale, j'espère que 2005 ne me contredira pas.

Mais le succès d'une revue ne tient pas que par ses auteurs. Au moment où certaines institutions reviennent sur le système élitiste que certains ont choisis, les revues thématiques de qualité, comme la nôtre, doivent se développer et il n'en tient qu'à vous.

À très bientôt

Henri Métivier

Président du comité de la revue

Agnès Henri

EDP Sciences 